University of Montana

ScholarWorks at University of Montana

Graduate Student Theses, Dissertations, \&

Professional Papers

1974

\title{
Effect of music on cooperative problem solving in children
}

Sanford Lee Chertock

The University of Montana

Follow this and additional works at: https://scholarworks.umt.edu/etd

Let us know how access to this document benefits you.

\section{Recommended Citation}

Chertock, Sanford Lee, "Effect of music on cooperative problem solving in children" (1974). Graduate Student Theses, Dissertations, \& Professional Papers. 2160.

https://scholarworks.umt.edu/etd/2160

This Thesis is brought to you for free and open access by the Graduate School at ScholarWorks at University of Montana. It has been accepted for inclusion in Graduate Student Theses, Dissertations, \& Professional Papers by an authorized administrator of ScholarWorks at University of Montana. For more information, please contact

scholarworks@mso.umt.edu. 
THE EFFECT OF MUSIC ON COOPERATIVE

PROBLEM SOLVING IN CHILDREN

\author{
by
}

Sanford L. Chertock

B.A., Ohio State University, 1971

\author{
Presented in partial fulfillment of the requirements \\ for the degree of \\ Master of Arts \\ UNIVERSITY OF MONTANA \\ 1974
}

Approved by:

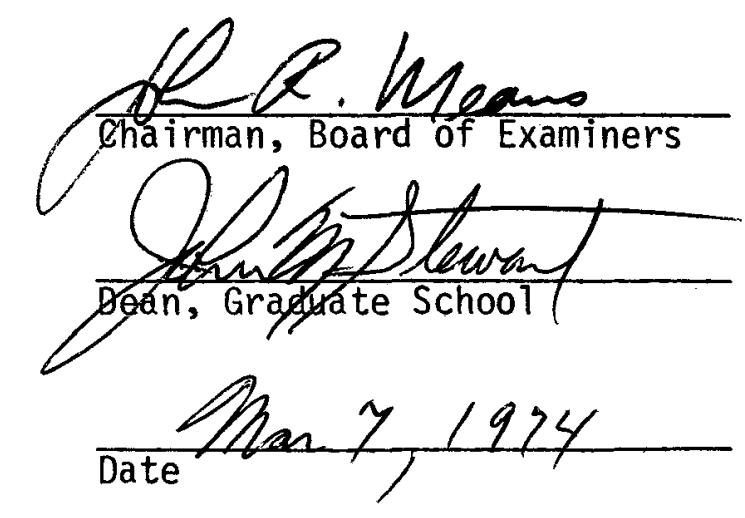


UMI Number: EP35633

All rights reserved

INFORMATION TO ALL USERS

The quality of this reproduction is dependent upon the quality of the copy submitted.

In the unlikely event that the author did not send a complete manuscript and there are missing pages, these will be noted. Also, if material had to be removed, a note will indicate the deletion.

UMI EP35633

Published by ProQuest LLC (2012). Copyright in the Dissertation held by the Author.

Microform Edition (C) ProQuest LLC.

All rights reserved. This work is protected against unauthorized copying under Title 17, United States Code

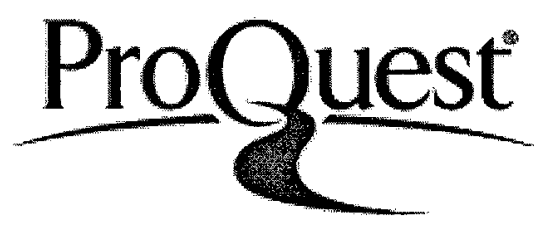

ProQuest LLC.

789 East Eisenhower Parkway

P.O. Box 1346

Ann Arbor, MI 48106 - 1346 
TABLE OF CONTENTS

Page

LIST OF TABLES ................... . . iv

LIST OF FIGURES ....................... v

CHAPTER

I. INTRODUCTION ................... 1

Cooperation and Competition in Children ...... 3

Measurement ................ 3

Cultural differences............ 13

Reinforcement .............. 16

Demographic factors ........... 20

Psychosocial factor ........... 22

Music and Children's Behavior.......... 23

Psychotherapeutic value ........... 23

Activity level............. 24

Social behavior ............. 25

Purpose ................... 26

Pilot Study................. 27

Theoretical Framework . . . . . . . . . . 29

Music perception as a conscious process . . . . 29

Attention and familiarity .......... 30

Definition of terms ........... 32

Hypotheses ............... 32

II. METHOD ................ 34

Subjects . . . . . . . . . . . . 34 
CHAPTER (continued)

Apparatus .................... 34

Procedure .............. . . . 37

Training of raters .......... . . 37

Unit of duration ................ 38

Procedural detail ............. 38

III. RESULTS ........................ 41

Inter-Rater Reliability . . . . . . . 444

IV. DISCUSSION .................... 47

Raters and Behavior Recording . . . . . . . 47

Subjects ..................... 50

Experimental Task . . . . . . . . . 5 52

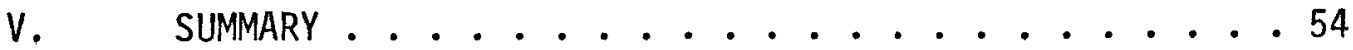

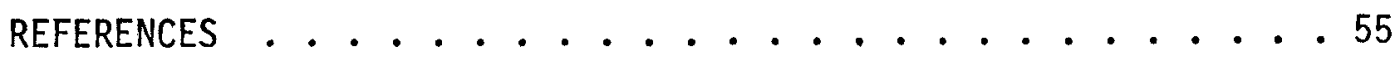

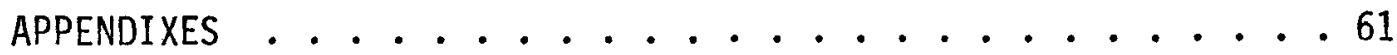

A Pilot Data Summary ............ 62

B Current Data Summary Including Residue Ratings . . 663

C Current Data Summary Excluding Residue Ratings . . 664

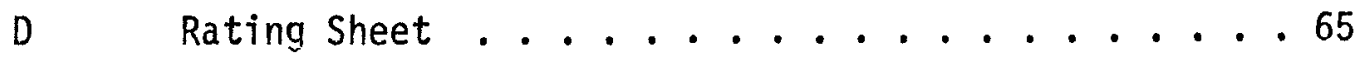




\section{LIST OF TABLES}

Table

Page

1 Summary of Analysis of Variance for

Cooperative Behavior I. . . . . . . . . 42

2 Summary of Analysis of Variance for

Cooperative Behavior II . . . . . . . . 4 42

3 Summary of Analysis of Variance for

Noncooperative Behavior I ......... 44 43

4 Summary of Analysis of Variance for

Noncooperative Behavior II . . . . . . . . 44 


\section{LIST OF FIGURES}

Figure

Page

1. Madsen Cooperation Board ............ 4

2. Marble Pull Game .............. 6

3. Circle Matrix Board ............ 8

4. Cooperation Box ................. 9

5. Diagram of Experimental Room ......... 35

6. Mean Group Performance Time as a Function of

Treatment Condition .......... 45 


\section{CHAPTER I}

\section{INTRODUCTION}

Man has produced music for centuries as a form of entertainment but only within the recent past has music's influence on the human organism been looked at in detail. Investigators, for the most part, have looked closest at the effects of music on three areas of functioning -- physiological, task completion, and therapeutic. Sugarman (1954), for example, observed that blood pressure decreased when slow tempo music was played. College students (Zimny and Weidenfeller, 1963), depressives and schizophrenics (Weidenfeller and Zimny, 1962), and five to twelve year old children (Zimny and Weidenfeller, 1962) all showed an increase in galvanic skin response (GSR) when exposed to music judged as exciting, and a GSR decrease while listening to calming music. Studies of task completion efficiency as a function of music, however, have produced differing results (Freeburne and Fleischer, 1952; Gatewood, 1921; Jensen, 1931). According to Smith (1947), music on the job facilitated completion of repetitive tasks, such as those found on assembly lines. Jacoby (1968) concluded that employees performing tasks relatively low in complexity, such as clerical services, experience more of a morale boost when hearing music at work than did those in technical, managerial, and administrative positions. 
Finally, the use of music as a psychotherapeutic aid has become increasingly popular. Greenberg and Fisher (1971) found that females, who took the Thematic Apperception and Draw-A-Person tests while hearing music they rated as exciting, told hostile stories and drew taller figures more often than when exposed to music they judged to be calming. There is even a periodical that reports therapeutic techniques for favorable altering of behavior through music -- the Journal of Music Therapy which began publishing in 1964.

Unfortunately, the closer look at music and human behavior has included few studies that explore changes in adult social behavior as a function of musical input in a non-therapeutic setting, and even fewer with respect to children. Measuring youngsters' activity level (Reiber, 1965) and controlling their activity level (Scott, 1970) have been accomplished with the use of music. Also, by employing music as a positive reinforcer, an educable mentally retarded child (Steele, 1968) and autistic children (Stevens and Clark, 1969) made noticeable strides in improving their social behavior. Blind children became less selfoccupied and more socially responsive after exposure to musical stimuli (Dryer and Dix, 1968). In order to further such experimental efforts in understanding how music affects children's social interaction, this investigation will attempt to determine if music influences the number of cooperative responses that six to nine year old children made while engaged in task completion activity. The topic is considered properly only after reviewing two larger related areas -- cooperation and competition in children, and the effects of music on behavior. 
Cooperation and Competition in Children

Measurement. In their classification of procedures employed by investigators of cooperative behavior, Hake and Vukelich (1972) divided all measurements of cooperative behavior into two types -choice and performance. Choice measurements deal with how cooperative solutions to tasks are chosen, while performance measurements are used in determining how these solutions are carried out. Most of the studies referred to will deal with performance measurements, since these have been most frequently reported.

Among the most objective and innovative methods for measuring cooperative and competitive behavior have been those developed by Madsen and his associates. Their game apparatus were designed so that jointly participating subjects ( $\underline{S} s$ ) respond in distinct, countable pulls and pushes which simplify behavioral observation and recording. Just as importantly, the apparatus were not all that different from games that children commonly play, like tug-of-war and checkers. Hence, $\underline{S}$ behavior while using these apparatus in the laboratory is likely to represent that found in naturalistic settings. Described below are four devices that these investigators have developed and used in their studies, as well as two other forms of performance measurement implemented by different researchers.

The Madsen Cooperation Board (Madsen, 1967; Madsen and Shapira, 1970; Miller and Thomas, 1972; Nelson and Madsen, 1970; Shapira, 1970; Shapira and Madsen, 1969) is an 18-inch square board with an eyelet in each corner (see figure 1). A separate string passes 


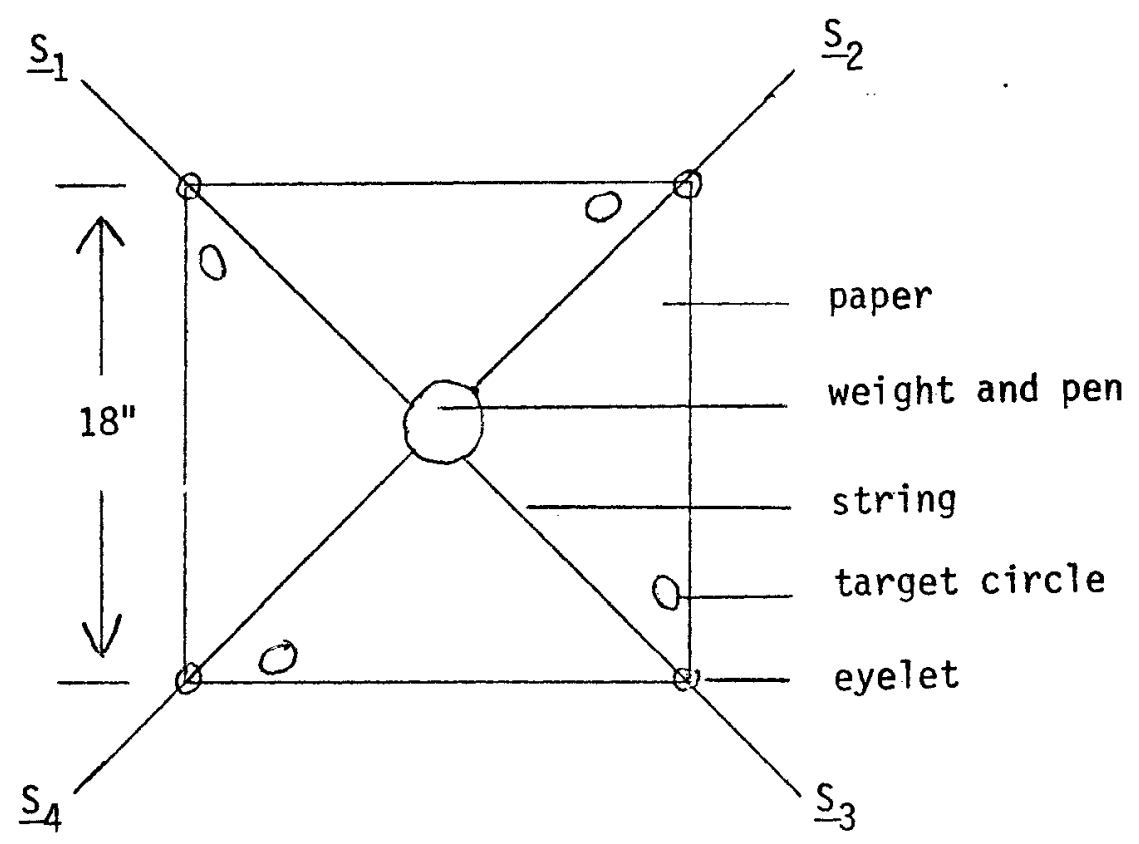

Figure 1. Madsen Cooperation Board 
through each eyelet, and all four strings are connected at the board's center to a weight holding a pen. The board is covered with paper, and a circle is drawn at each corner of the paper where a $\underline{S}$ sits. A $\underline{S}$ receives a toy every time he pulls the pen across the circle at his corner with his string. If the $\underline{S}^{\prime} s$ pen line deviates one inch or less from a direct path between the board's center and his corner circle, the response is judged to be cooperative. Deviation greater than one inch is scored as competitive. The cooperative solution is for $\underline{S} s$ to alternate pen pulls toward their individual circles on different trials so that all ss can earn a nearly equal number of toys. Simultaneously pulling two or more strings moves the pen across none of the four circles.

Madsen used a second technique which he called the Marble Pull Game (Madsen, 1971) (see figure 2). A receptacle containing a marble is placed in the center of a rectangular table that has a recessed cup at either end. One string is attached to each end of the receptacle, and each $\underline{S}$ pulls his string to bring the holder over the cup at his end of the table, whereupon the marble is released into the cup and $\underline{S}$ retains the marble. However, when the strings are pulled simultaneously, the receptacle breaks apart and the marble rolls into a groove, signifying no reward for either $\underline{S}$. Again, in order for $\underline{S} s$ to receive maximal compensation, they must devise a system of alternate trial reward taking. The number of marbles $\underline{S} s$ accumulate at the end of ten trials is the measure of cooperation displayed. 


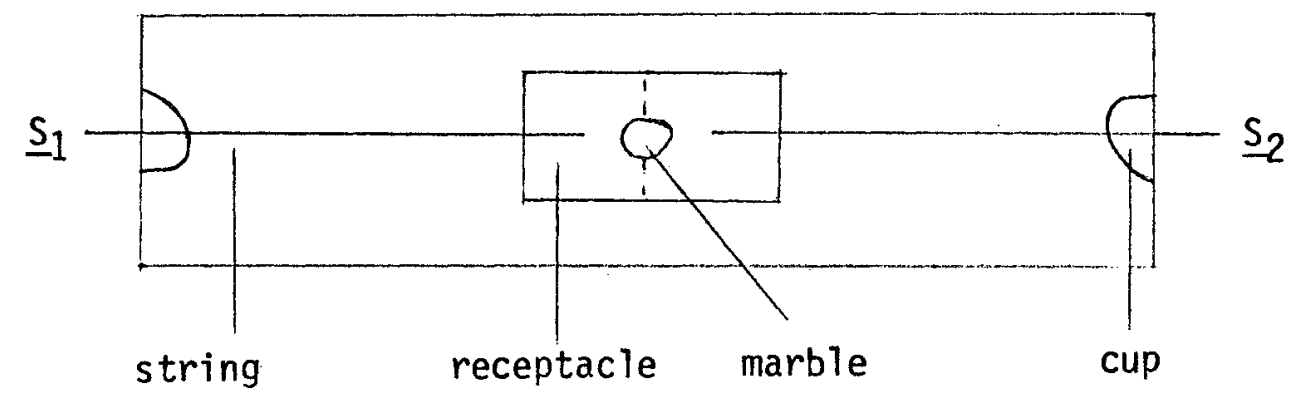

Figure 2. Marble Pull Game 
Children who are not fond of pen or marble pulling might enjoy the Circle Matrix Board -- a third approach the Madsen group has taken to measure competition and cooperation (Kagan and Madsen, 1971, 1972). Ss sit on opposite sides of a square board containing seven columns and seven rows of equidistant circles connected by one-inch lines (see figure 3). After a marker is placed in the center circle (spotted circle in figure 3), Ss take turns moving to adjacent circles. A trial ends when the marker lands on the striped circle, whereupon $\underline{S}_{1}$ receives a toy, or when it lands on the cross-hatched circle, signifying a toy for $\underline{S}_{2}$. Both $\underline{S}$ s can only move to one adjacent circle per turn, and a total of twenty turns (moves) is allowed per trial. Resulting data is analyzable into the number of toys won, the number of moves made, and the types of moves made -- e.g., cooperative, indicated by reaching the cross-hatched or striped circle in the first three moves of a trial, or nonconflicting, indicated by movement outside the path outlined by dotted lines in figure 3 .

Still another apparatus that Kagan and Madsen describe is their Copperation Box (Kagan and Madsen, 1972, see figure 4). The box can only be opened by each of the two Ss using both hands to simultaneously unfasten all four latches. The box either contains two identical toys, one of which is given to each $\underline{S}$ after the $\underline{S s}$ open the box, or just one toy to be taken by one $\underline{S}$, openly designated as the recipient before the box is opened. In either case, the dependent variable is the amount of time required by $\underline{S}$ s to open the box. 
S?

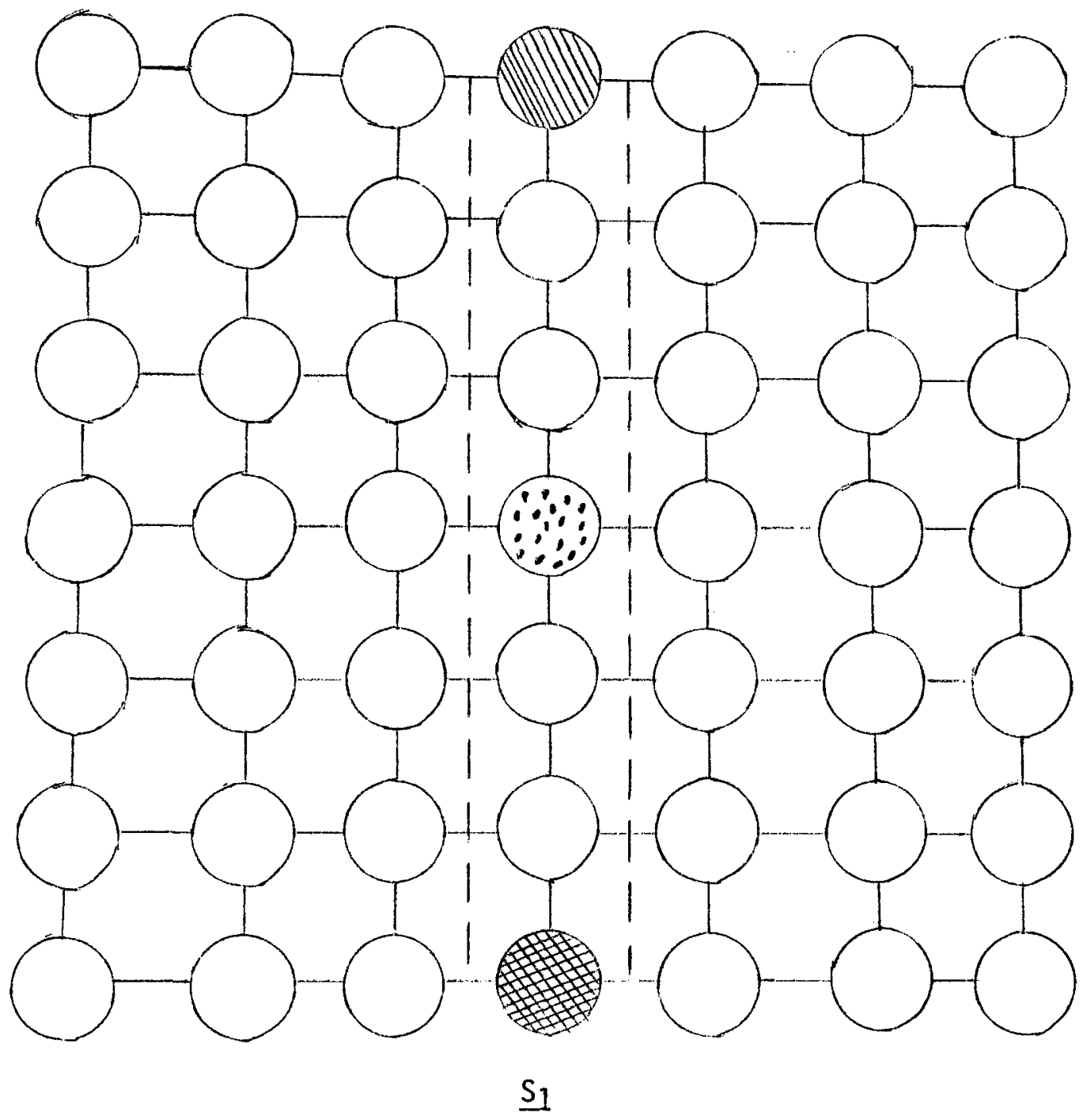

Figure 3. Circle Matrix Board 


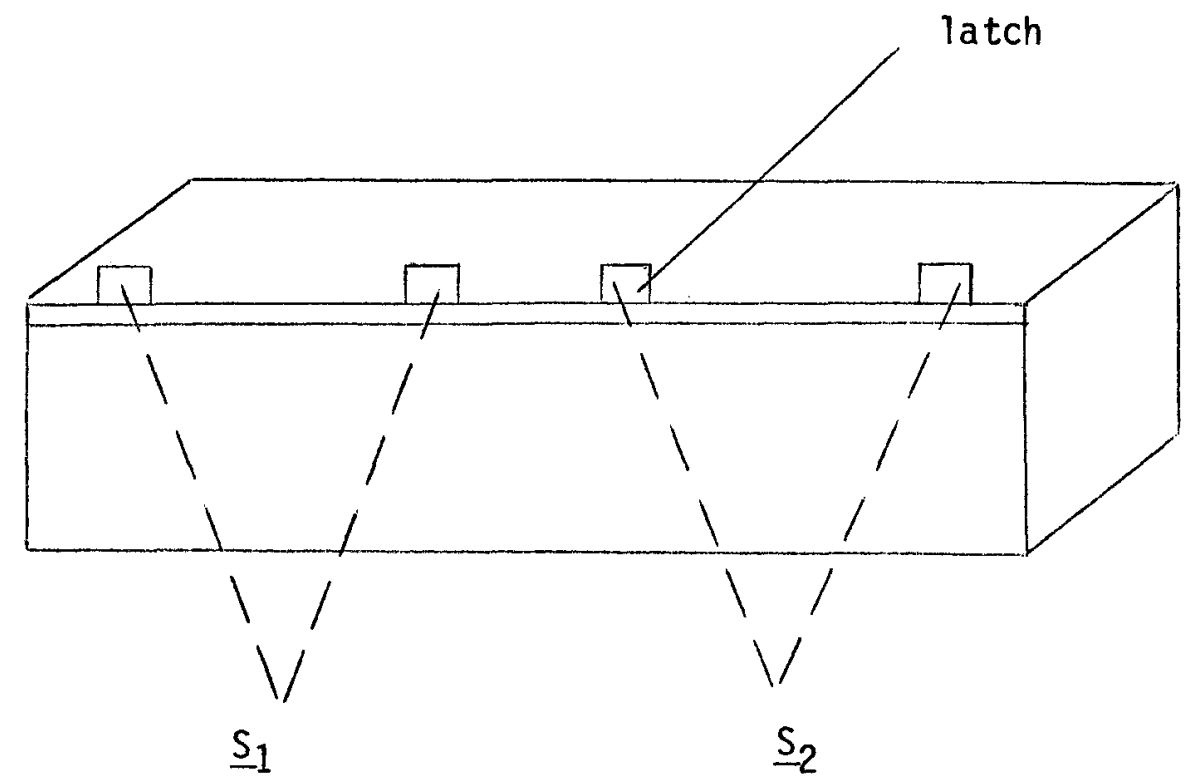

Figure 4. Cooperation Box 
Other researches have constructed elaborate electrical mechanisms. For instance, in their study on maintaining copperative group responses, Mithaug and Burgess (1968) used three $\underline{S}$ instruments, each composed of fourteen piano-like keys, two lights, and an electromechanical counter which recorded the number of responses $\underline{S}$ s made. On the experimenter's (E's) desk were an electromechanical counter for each $\underline{S}$ s instrument, a group of switches for illuminating screen lights corresponding to those on the $\underline{S}$ instruments, and another set of switches controlling the $\underline{S}$ instrument lights. Wasik, Senn, and Epanchin (1969) used a switch and light apparatus when viewing cooperation and sharing as a function of race, as did McClintock and Nuttin (1969) in their work on motivation to cooperate as a function of age. In addition, devices have been constructed so that reinforcement is mechanically delivered by $\underline{S}$ pressing telegraph keys (Brotsky and Thomas, 1967), placing styli into holes (Azrin and Lindsley, 1956) and pulling levers (Weingold and Webster, 1964).

The studies cited in the above paragraph all measured cooperation in terms of number of button pushes, lever pulls, or other contrived means of responding. Altman (1971) claimed that, after acquiring a cooperative response set by pulling levers on his electrical apparatus, $\underline{S}$ showed a subsequent increase in the number of associative responses and a descrease in the number of hostile responses during a free play session as compared to baseline rates taken before $\underline{S}$ performed on the apparatus. Unfortunately, his 
criterion for scoring a response as associative -- " . . When children seem aware of a common interest, activity, or goa] . . [A]tman, 1971 , p. 13]" - - was not specific enough to precisly compare pre- and postexperimental associative behaviors. A post-experimental decline in hostility toward peers might simply reflect a state of satiation following reinforcement obtained during the experiment. However, an increase in friendly conversation with peers would be a more objective sign of increased friendly social interaction. Altman reported no significant increase in conversation, stating that conversation could not be expected to increase because it was not part of the experimental reinforcement contingencies. This and other tasks in which children perform on electrically-rigged mechanisms, though interesting, must be regarded critically in view of most children's daily social interaction settings which, presumably, do not include cooperative ventures on such devices. Approximation to the actual environment must be as close as possible (Wodarski, Hamblin, Buckholdt, and Ferritor, i972).

Finally, responses can be measured by raters who are specially trained to observe and record behaviors of interest, regardless of the type of apparatus, or even if none is used. Hannah (1970) adopted this method to measure classroom competition and cooperation as a function of task similarity between $\underline{S}$ groups. In another study, under conditions where two Ss had to share one crayon, raters recorded the number of times each $\underline{S}$ asked the other for the crayon and 
the time intervals each S kept it (Staub and Sherk, 1970). Raters usually observe and record within equal, successive time intervals -- e.g., ten seconds of observing followed by ten seconds of recording. This time sampling observation technique has been successful in educational psychology research, where Bushe11, Wrobel, and Michaelis (1968) used it to record preschoolers' study behaviors. Meichenbaum, Bowers, and Ross (1969) applied it to assess the effects of teacher expectancy in institutionalized female adolescent offenders in the classroom, and Hops (1971) instructed teachers in using it to measure task and non-task oriented behavior. Wodarski, et al., (1972) recently applied it to measuring changes in cooperative behavior as a function of the individual to group reinforcement ratio used. Once behaviors to be observed have been narrowly defined and raters trained to reliably observe and record, the method is objective and produces data suitable for statistical analysis.

These, then are the principal forms of measurement which investigators have used to assess cooperative responding in children. Clearly, the four developed by the Madsen group -- the Madsen Cooperation Board, Marble Pull Game, Circle Matrix Board, and Cooperation Box -- are desirable measures of cooperative behavior. $\underline{S}$ responses are readily observable, scorable, and most importantly, objectively classified as either cooperative or noncooperative, not somewhere in between. In fact, the Madsen Cooperation Board even produces its own response record on the paper covering it. While somewhat less 
precise than these devices, the use of timed observations allows E to chart a greater bandwith of $\underline{S} s^{\prime}$ cooperative behaviors, and in this sense provides an objective, more well-rounded picture of $\underline{S}$ behavior patterns. Since the effect of music on cooperative problem solving has been scantily researched at best, it was decided to use this latter method for the proposed study in order to determine if gross motor and verbal cooperative responses, occurring when music is present, are different from those when music is absent.

Cultural differences. Despite the spread of American technology to other nations since World War II, the high degree of competition between individuals that helped to shaped this technology is apparently not being transmitted to children of certain countries. One instance is Mexican children. Madsen (1971) found Mexican children to be far less competitive than Anglo-American children, even when the latter were given an explicitly cooperative instructional set. Kagan and Madsen (1971) observed that Mexican Ss made fewer competitive responses than Mexican-American $\underline{S} s$, who in turn made fewer competitive responses than Ang10-American $\underline{S} s$ under each of four different instructional sets. In a related study (Kagan and Madsen, 1972), a distinction was made between motivation to cooperate and conflict. American children were just as highly motivated to cooperate in helping a peer win a toy by unlatching the Cooperation Box as were Mexican children. Yet, when American Ss could not win a toy when using the Circle Matrix Board, they competitively prevented an 
opponent from keeping one that $\underline{E}$ had given to the opponent twice as often as Mexican $\underline{S} s$ did. By refusing to make competitive moves and prevent opponents from winning their toys, Mexican Ss consistently avoided conflict. The authors concluded that, while there was no evidence for a difference between American and Mexican cultures in notivation to cooperate, children of both cultures are systematically irrational products of different conflict ideologies -- the American, which extols conflict, and the Mexican, which emphasizes conflict avoidance. In additional support of this conclusion, Afroand Mexican-American children have also been shown to outrank Mexican village children in degree of competitiveness (Madsen and Shapira, 1970).

Belgian children also differ from American children on the cooperation-competition continuum. According to McClintock and Nuttin (1969), until the sixth grade, American Ss chose to compete while playing a Maximizing Difference Game more often than did the Flemish. Interesting to note, however, is the higher competitiveness that children of both nationalities displayed when they were appraised of their own and their opponent's cumulative score. It appears, then, the American children do not hold a monopoly on the desire to surpass, or at least maintain an equal footing with their peers' task performance. Yet, it must be noted that teachers, in both cultures, chose only their brighter students for the experiment, and from these Es selected those who thoroughly understood the task. Hence, the results do not necessarily hold for both cultures in 
general, as the authors seem to indicate.

Researchers have even found differences at the subcultural level. In one experiment, Israeli urban and kibbutz children had to choose between earning individual reward and contributing to their group's chances to winning a game with cards (Shapira, 1970). Kibbutz children contributed more cards to their group than did city $\underline{\text { Ss. }}$ Shapira and Madsen (1969) found that, while both city and kibbutz Ss cooperated under a group reward condition, only the kibbutz Ss maintained adaptive cooperation when the group reward contingency was switched to individual reward. The authors hypothesize that, because the kibbutz life style of group living is potentially far more competitive than that of city children, kibbutz children adopt strong cooperative tendencies so that the group can function properly. In another subculture study, Madsen (1967) found that urban middle class Mexican children show higher competitive motivation than their urban poor and Indian village counterparts. Madsen felt that these results could be accounted for by the fact that the prime concern of a family living in poverty is to obtain basic necessities, like food, and a child is likely to be discouraged from competing with other family members for more than his share of familial reserves. Generally, then, work with subcultures indicates that urban children have a greater propensity for competition than nonurbans do. A final example further supports this finding. After observing and recording behavior from Blackfoot Indian and urban $\underline{S}$ s in Alberta, Miller and Thomas (1972) found that urban Ss could not inhibit 
competitiveness under an individual reward condition as well as Indian Ss could when performing on the Madsen Cooperation Board. Urban $\underline{S}$ s developed such an unyielding competitive strategy that they of ten complained that their hands hurt them because they were pulling the strings too hard.

The above studies seem methodologically sound. Groups in compared cultures were equated for sex and age, equipment and instructional sets were constant across treatments, and control was present in the form of one cultural group serving as the control for the other. Es pointed to cultural background differences as possible explanations for obtained performance differences, yet judicially did not label them as causative factors in the absence of experimental evidence.

Reinforcement. The studies cited below have found cooperative behavior to be maintained by effective reinforcement. Variables which determine whether a given reinforcer is effective have been the type of reinforcement administered and upon whose behavior it is contingent -- that of the individual or group.

That cooperative responses are maintained by reinforcement was shown in an early study by Azrin and Lindsley (1956). Ss emitted far less cooperative behavior during an extinction period than during a preceding acquisition and succeeding reinforcement period. The authors claimed that the decline in cooperation during extinction was due specifically to the absence of reinforcement, not to fatigue 
or satiation. Further investigating cooperation curtailment during periods of extinction, Weingold and Webster (1964) first operantly conditioned cooperative responses in male dyads by regulating reinforcement delivery via a control panel. Then, half the dyads underwent punishment, in which one dyad member lost previously acquired M\&M candies when he now responded cooperatively, while the other member continued to receive M\&Ms for cooperative responding. The other dyad half underwent extinction, where reinforcement was simply withheld from one dyad member for responding cooperatively while the other member continued to receive M\&Ms for cooperative responding. As a result, punished dyads dropped their rate of cooperative responding below that established during acquisition, while dyads on extinction did not. Apparently, cooperative responding can be extinguished far more quickly by taking away material rewards already earned by it than by withholding material rewards yet to be given in return for it. The question of what is an effective reinforcer of cooperative behavior led Fischer (1963) to study the value of using material reward vs. verbal praise as a reinforcer. Half of the $\underline{S} s$ who shared marbles with a fellow $\underline{S}$ received gum; the other half, verbal praise. Ot those who reached the sharing acquisition criterion, $85 \%$ had been reinforced with gum, while $15 \%$ had been verbally praised, suggesting that material reinforcement may be superior to verbal in training 42-57 month old children to share. In defense of verbal reinforcement, however, other experimenters showed how a child's predominantly non-social 
behavior toward peers could be verbally modified so that she displayed more cooperative play responses toward children than attention seeking responses toward adults (A1len, Hart, Buel1, Harris, and Wolf, 1964). After making teacher praise contingent upon successively closer proximity to and greater interaction with fellow preschoolers, these experimenters noted an increase in $\underline{S}$ 's cooperative peer interactions from $10 \%$ to $60 \%$ of a 11 her interaction observed during daily rating periods. Post checks on $\underline{S}^{\prime} s$ behavior for four weeks showed an average peer interaction rate of $54 \%$ of a 11 her observed interactions. In a similar study, noncontingent verbal reinforcement from teachers did not produce an increase in cooperative play with peers, while contingent verbal reinforcement did (Hart, Reynolds, Baer, Brawley, and Harris, 1968).

Not all studies have separated the effects of diffferent forms of reinforcement. In the Miller and Thomas (1972) study, E combined material and verbal reinforcement in one of the two experiments reported. Group cooperation and individual competition were both reinforced verbally each time $\underline{E}$ announced when a set of circles (group condition) or a $\underline{S}^{\prime} s$ circle (individual condition) had been marked on the Madsen Cooperation Board. Additionally, they received toys for this verbally reinforced behavior. It is possible that Indian and urban Ss may have shown more or less cooperative responding had only one type of reinforcement been provided, depending on their past reinforcement histories. Conceivably, the use of a mechanical reinforcement deliverer to provide material reinforcement for each 
individual or group of circles crossed would have eliminated the need for simultaneous verbal cues.

Just as important in predicting the effectiveness of a reinforcer is knowing who will be rewarded for cooperating -- the individual or the group in which the individual participates. As previously noted, cooperation in urban Canadian (Miller and Thomas, 1972) and Israeli (Shapira and Madsen, 1969) children broke down after an individual reinforcement contingency replaced the rewarding of group behavior. Similar results exist for American Negroes and Caucasians (Nelson and Madsen, 1969). Mithaug (1969) found that when Ss were able to distinguish between payoffs for participating in individual and group tasks, and when higher reward was given for group than individual performance, Ss chose to participate in the group task more often than in the individual task. In another attempt to determine whether different amounts of individual and group reward contingencies could produce corresponding different amounts of cooperation, one group of investigators rewarded $\underline{S}$ s for correctly solved arithmetic problems with token dollar bills and coins under four different group-individual contingencies: $100 \%$ group, $67 \%$ group $/ 33 \%$ individual, $33 \%$ group $/ 67 \%$ individual, and 100\% individual (Wodarski, et al., 1972). That is to say, under the $100 \%$ group contingency, Ss received dollar tokens for the average number of correctly solved problems worked by those $\underline{S} s$ with the bottom four performance score totals. Under the $100 \%$ individual contingency, each $\underline{S}$ received dollar tokens for his own correctly solved problems. Under the mixed contingencies, a 
$\underline{S}$ received either 67 cents for each problem he correctly solved plus 37 cents for the average of the group's bottom four performances, or vice-versa. The results showed a linear relationship between the percentage of group reward contingency and the amount of cooperative behavior displayed. The more group goal behavior was rewarded at the expense of individual goal behavior, the more cooperative responses were emitted.

Demographic factors. Another perspective from which to consider children's cooperative and competitive behavior concerns the influence of demographic variables. While studies of this topic are not abundant, preliminary work has been done on the variables of age, sex, race, and socioeconomic status (SES).

Most studies looking at cooperation as a function of age have reported a greater incidence of cooperation in younger children and competition in older ones. Owens (1969) observed an increase in competition with increasing age in $\underline{S}$ sho, after dropping marbles in a box as quickly as possible while alone, were exposed to a marble dropping machine while performing the same task. After comparing Circle Matrix Board performance of 4-5 year olds with that of 7-9 year olds, Kagan and Madsen (1971) found the younger children to be more cooperative in all three cultures sampled -- Mexican, MexicanAmerican, and Anglo-American. In the McClintock and Nuttin (1969) study, competition was greater in older children of both Flemish and American cultures. Other studies have demonstrated how $\underline{S}$ around 
age four years cooperated when only cooperative cues were present (Nelson, 1970; Nelson and Madsen, 1969), as did older Ss up to age ten years (Nelson and Madsen, 1969). Yet when only competitive cues were present, younger $\underline{S} s$ were far less competitive than older $\underline{S} s$, suggesting that learning to compete when competition is the only adaptive response possible is a developmental phenomenon. An exception to the older child-more competition trend is an experiment by Handlon and Gross (1959) in which older children were more likely to give a partner more than half of an unequally divisible number of objects than were younger children.

The extent to which sex determines the probability of establishing a cooperative response has not been determined. Though McKee and Leader (1955) and Shapira (1970) found boys to be more cooperative than girls, other researchers reported only significant interactions between sex and other factors, like age (Nelson, 1970), culture (Miller and Thomas, 1972), and environment (Doland and Adelberg, 1967). Many more experimenters have simply pre-matched $\underline{\text { Ss }}$ into like-sex dyads to control for sex differences (Azrin and Lindsley, 1956; Luchins and Luchins, 1957; MCClintock and Nuttin, 1969; McKee and Leader, 1955; Madsen, 1971; Peters and Torrence, 1972; Wasik, et al., 1969; Weingold and Webster, 1964), thus avoiding the problem altogether. Other findings tell of sex differences in neither cooperation (Brotsky and Thomas, 1967; Kagan and Madsen, 1971) nor sharing behavior (Handlon and Gross, 1959; 
Ugurel-Semin, 1952). Hence, a consistent relationship between sex and cooperative behavior has not been confirmed, and further research is needed to determine the precise conditions under which sex may be an influencing factor.

The results of studies on how race and SES influence cooperation are just as inconclusive. Harford and Cutter (1966) found Negro males to be less cooperative than Negro females, with Caucasian males and females falling somewhere in between. Nelson and Madsen (1969) found no race difference. McKee and Leader (1955) concluded that low SES children tend to compete more than those from the upper middle class, while Nelson and Madsen (1969) found no differences between middle class Ss and Head Start enrollees in amount of cooperation displayed. Again, only further inquiry into the specificity of these variables will help to explain seemingly contradictory results.

Psychosocial factor. A final variable to consider under children's cooperative and competitive behavior is how the peers to whom such behavior is directed are perceived. In one study on cooperative altruism, Ss gave more marbles to absent children, whose pictures they saw and about whom fabricated statements of reciprocity they heard from $\underline{E}$, than to other nonexistent children about whom they were told and shown nothing (Presbie and Kapareff, 1970). In another study, Wright (1942) found a positive relationship between how willing a child was to give away a favorite toy to a friend, and how generous he believed his friend to be. Zwier (1964) noted that elementary 
school $\underline{S} s$ chose to talk most often with peers who they and other $\underline{S} s$ rated as being similar to themselves in social power, intelligence, and popularity. When looking at cooperation as a function of sociometric scores, however, Katz (1965) observed the greatest amount of cooperation in $\underline{S}$ pairs composed of one $\underline{S}$ who had received mostly high ratings from peers on the Syracuse Scales of Social Ratings, and another $\underline{S}$ who had received mostly low peer ratings. Apparently, two children of similar social attractiveness do not necessarily cooperate well with each other.

Music and Children's Behavior

Psychotherapeutic value. Diephouse (1968) wrote one of the few reviews of studies on how music affects children's behavior in a psychotherapeutic setting. Regarding physiology, he said there was evidence to indicate that slow, softly played music contributes to the parasympathetic autonomic nervous system's rebuilding of body tissue. Looking at communicative behavior, he noted that music is a language which children do not have to learn. Neither are they burdened with attached social connotations so frequently a part of the spoken word. Weigle (1959) showed how children who never spoke a complete sentence began singing entire songs during singing sessions in group therapy. Diephouse (1968) referred to the case of an elective mute who, after several music therapy sessions, began communicating with the therapist by singing. Properly used, music is valuable in child psychotherapy because 
it can serve to make the therapeutic setting more attractive, hence be a source of motivation to come to therapy, and can encourage children to be responsive to other forms of therapy as well (Diephouse, 1968).

Activity level. Music has also been found to affect children's activity leve1. Rieber (1965) connected automatic counters to six toys in a playroom, and observed the total number of turns, presses, and foot rotations $\underline{S} s$ made on the toys when background music was alternately present and absent. Activity rates were higher during music intervals, and higher for fast than slow musical selections. Scott (1970) gave arithmetic problems to four hyperactive boys under four conditions: (1) in an open area resembling a classroom; in an area identical to the first condition except for background music being played at a normal listening level; (3) in three-sided booths which reduced noise and social interaction levels; (4) in booths identical to the third condition except for background music being played. Three of the Ss correctly solved the most problems under condition (2), indicating that background music may promote better academic performance in hyperactive children.

A critical look at these two studies reveals good measuring technique in both, but a difference in control over systematic bias. Rieber's (1965) dependent variable was automatically recorded, and he controlled for music presentation with a no music condition, the music and no music intervals being of equal duration. To eliminate 
systematic bias, however, he should have randomized the order in which the music and no music periods were presented, instead of administering them in the same no music - music - no music - music sequence. Scott's (1970) dependent variable was also objectively, thoughnot automatically, measured by simply counting the number of correctly solved arithmetic problems under each condition. Yet, unlike Rieber, he effectively controlled for extraneous factors through random presentation of the arithmetic problems and systematic rotation of the experimental conditions for all Ss.

Social behavior. Still another way in which music has influenced children's behavior has been to increase their willingness to socially interact. After eighteen sessions in music therapy, four of five autistic boys showed an increase in some prosocial behaviors, such that three of the five were placed in public school (Stevens and Clark, 1969). After musical sessions in therapy with an initially self-centered, aggressive eight year old retarded boy, Steele (1968) noted that the boy's aggressive behaviors disappeared. She also found that the amount of time he spent in cooperative behavior within a 6 -foot radius of the therapist increased from 4 to 25 minutes during a 30-minute session, and that this cooperative set generalized to a special education class. Through the use of music, the therapist claimed that she was able to establish verbal control over S's social responses and channel much of his self-directed behavior into otherdirected, cooperative activity directed at her. 
Though her procedure was reported as effective, there is no sure way of telling if music alone was responsible for the increase in social responsiveness that Steele (1968) observed, due to lack of environmental control. Different environmental stimuli, ranging from therapist singing to introducing drums and a record player, were incorporated at various treatment stages in addition to the background music already present. Certainly, any claims that background music singly altered uncooperative behavior are unjustified. Comparisons between baseline and treatment data could have been valid only if environmental constancy had prevailed.

\section{Purpose}

The purpose of this investigation was to determine if music influences the number of cooperative interactions that six to nine year old children make while engaged in task-solving activity. To date, studies of children's cooperative behavior have either ignored the music variable, or else have implemented it in a therpeutic setting in order to produce a desired behavioral change. Few researchers have observed the effects of music on cooperative responding, in a non-therapeutic setting, where individual reinforcement is contingent upon cooperation between group members. It is from this type of operational framework that changes in $S_{s}$ ' behavior were noted when background music was present or absent.

The problem of establishing cooperative tendencies during childhood seems highly relevant when considering the increased amount of 
social contact that today's children will have in tomorrow's world of improved communication systems and increased social stimulus input. While some degree of classroom competition may be desirable to spur a child on to perform to the limit of his abilities, it is at least equally important to establish a strong basis for emitting cooperative responses when they are called for. The possibility of establishing cooperative behavior through music in the classroom is a line of subsequent inquiry that might follow the present, more basic one of observation.

\section{Pilot Study}

During August, 1973, the author conducted a pilot study to test for differential effects of harmonious and non-harmonious background music on the number of cooperative responses displayed by $35 \mathrm{six}$ to nine year old children. A total of five $\underline{S}$ groups, with seven $\underline{S} s$ per group, were run. The task and experimental methodology of this initial study were identical to those of the current work (see Chapter II), with the exception of the treatment conditions. In the pilot study, these were the playing of harmonious music $(H)$, nonharmonious music $(\mathrm{NH})$, voices of three adults reading aloud simultaneously from different textbooks (PT), and no sound (NS).

Procedural refinements were made midway through the study, resulting in improved $\underline{S}$ performance and a decrease in time needed to run each $\underline{S}$ group. Specifically, the number of cards given to each $\underline{S}$ was reduced from fourteen to eight, $\underline{S}$ s received an increased number of M\&Ms after 
completing each of the four treatments, and the instructional set was modified to include an explicit directive for trading cards with one's neighbors. In addition, the rating form on which children's behaviors were recorded was revised once during the pilot study, and a second time before starting the current study.

The data from groups 3, 4, and 5 were analyzed in a two-factor mixed design with repeated measures on one factor. This design was chosen so that each $\underline{S}$ could be randomly assigned to one level of the treatment factor. A summary of pilot data may be found in Appendix A. No total group performance time was obtained for the first treatment administered to Groups 1 and 2, since they did not complete the task within the ten minutes allowed for rating (see Chapter III). Therefore, results for these groups are given as the total number of cooperative and noncooperative responses recorded under all treatments. Data for groups 3, 4, and 5 are reported as the number of cooperative and noncooperative responses displayed per minute. Mean interrater reliability was .82 .

Upon initial analysis, the treatment effect for cooperative behavior of groups 3,4 , and 5 was significant at the .06 level, while variance accounted for by the noncooperative group factor, for these same three groups, was significant at the .005 level. Unfortunately, the credibility of these findings became questionable after the presence of three types of variable confounding became clear: group $X$ trial, group $X$ treatment, and group $X$ treatment sequence. 
In perspective, the pilot study pointed emphatically to the need for treatment presentation that would not allow a practice effect to occur. Though practice effects might have been somewhat more analyzable had a replicated Latin Square Design been used, the author decided to substitute a simple one-way analysis of variance design, in place of a repeated measure design, for the final study. In this manner, each $\underline{S}$ group would only receive one treatment, and the above confounding effects could be substantially reduced, if not eliminated.

\section{Theoretical Framework}

Music perception as a conscious process. In his discussion of music as a conscious, phenomenological entity, Pike (1967b) argued that music is labeled warm, harsh, active, or lethargic not because one concentrates on individual tones, but rather because he perceives conbinations of individual tones into tonal gestalten (wholes), like phrases and motives. "These formations have their own properties of pitch relationships, rhythm, tempo, and dynamics [Pike, 1967a, p. 317]." For example, though the different pitches of a vibrato are not perceived separately, the center tone around which the pitches vary is perceived. One consciously hears the beginning and end of a glissando, and just enough of the intervening tones for identification purposes. Transitive chords are consciously listened for as part of " . . the total gestalt of 'preparation-suspension-resolution [Pike, 1967b, p. 396]" so often found in classical and popular melodies. The point is that feelings or moods aroused by music are not necessarily governed 
by the unconscious, since they can be traced to conscious operations the individual performs while listening to the music.

The conscious perception of music, claimed Pike (1967b), is immediate. One does not first perceive the change from a diminished or augmented major chord to a major sixth chord, and then experience a subsequent transition from a tense to a relaxed internal state. The two occur together, as though they were part of the same phenomenon. That music can produce a heightened physiological state has already been demonstrated (Weidenfeller and Zimny, 1962; Zimny and Weidenfeller, 1962, 1963). Since a heightened physiological state is often accompanied by observable changes in behavior, hearing music may be indirectly, yet immediately responsible for concomitant behavioral changes.

Attention and familiarity. Lathom (1971) noted that, according to information theory, if the probability of accurately predicting an upcoming event is high, that event contributes little or no new information, and, therefore, is not vigorously attended to. Since the successive notes of a musical theme frequently fall into this category, she concluded that, unless he listens to music containing some unexpected elements, the music listener can simultaneously attend to other, Jess redundant activities.

Distraction may also be involved. According to Mussulman (1974), if the music contains no sudden, unexpected changes in such variables as density, volume, timbre, and tempo, it is not unreasonable to anticipate 
an increase in work output while the music is played. On the other hand, some composers deliberately include senorious variation to attract the Tistener's attention. Mussulman called the former type of music "mood music," and the latter "art music." It would seem, therefore, that, when compared to no background sound, or to one other than music, mood music would facilitate task achievement by directing one's attentive powers to the task at hand, while art music would not.

A third theoretical viewpoint takes stimulation to be of central importance. By and large, Western society children are frequently exposed to a variety of music, artistic and mood, via mass media and in vivo. Since a good deal of this exposure is voluntary, e.g., turning on radio, TV, record player, apparently the act of listening to music acquires secondary reinforcing properties at an early age. Children often observe how adults use the well-practiced habit of listening to music to stimulate themselves while performing solitary, routine activities like cooking, studying, and grocery shopping. Perhaps, after a requisite amount of exposure, children acquire the set "music can be stimulating." They probably do not differentiate between mood and art music in terms of stimulating value, and could be expected to show increased task performance efficiency when either is played, as opposed to when silence or some other form of background sound is present. 
Definition of terms. In this investigation, the term cooperation is operationally defined as verbal and/or physical action taken by a $\underline{S}$, while receiving assistance from or giving assistance to another $\underline{S}$, in order to complete the assigned group task. Specific cooperative and noncooperative behaviors will be defined as those appearing on specially prepared rating forms used by trained observers of children's behavior (see Appendix D). Mood music, for the purposes of this experiment, is music which has no sudden, distractable alteration of volume, tempo, density, or timbre. Art music does contain such an alteration in one or more of these variables. Mood music and art music selections used were, respectively, "The Waltz You Saved for Me," played by the Living Strings, and Takemitsu's "November Steps."

Hypotheses. In light of the above discussion, it is hypothesized that music will differentially affect children engaged in a cooperative problem solving task, such that they will display more cooperative responses per minute while music is present -- art or mood -than when exposed to a tape recording of people talking or no sound. The reasoning here is that, because of prior association with task performance in adults, senorious input should serve to stimulate the children to deal with the task at hand. Since completion of this task necessitates cooperative responding, cooperative responses should be more frequent when music is present than when it is absent. Similarly, noncooperative responses -- i.e., all responses not rated as cooperative -- should be greater under no music than under music conditions. 
Additionally, the number of cooperative responses per minute is hypothesized to be greater, and noncooperative responses fewer, when children hear mood music as compared with art music. Mood music should contain less distractable elements than art music, and consequently allow more attention to be directed toward successful task completion, which, again, necessarily involves cooperative responding. 


\section{CHAPTER II}

METHOD

\section{Subjects}

Ss were 64 male and 76 female children, ranging in age from 6 years, 3 months to 9 years, 2 months. The mean age was 8.12 years. Approximately 41 percent were students in School District No. 1 of Missoula, Montana, while 59 percent attended Missoula's St. Anthony Religious Education Center. Children were allowed to participate after their parents signed a permission sheet. Parents of public school children were individually phoned, assured of the study's legitimacy, and requested to bring their children to the University of Montana's Clinical Psychology Center at one of the times indicated on the permission sheet. Parents of St. Anthony pupils were not contacted by phone because their children participated at the school during school hours.

Seven children were randomly assigned to a group. Each group received only one treatment, and there was a total of five groups, for each of four treatment conditions that received the same treatment.

\section{Apparatus}

The first nine $\underline{S}$ groups were run in a room of the Clinical Psychology Center (see figure 5). Positions occupied by raters and 


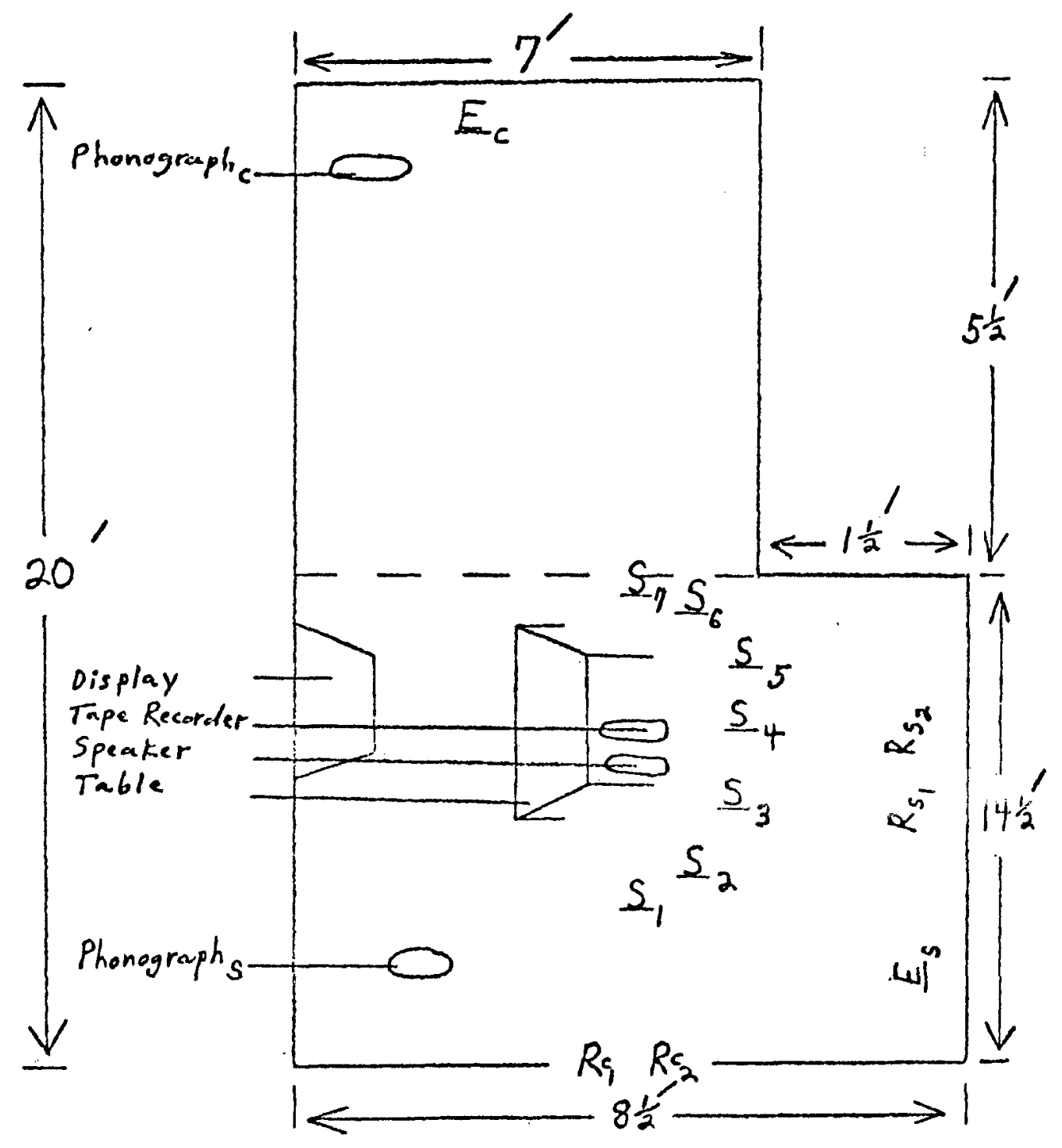

Figure 5. Diagram of Experimental Room 
E were, respectively, $R_{1}, R_{2}$, and $\underline{E}_{c}$. The remaining 11 groups participated at St. Anthony in a schoolroom where apparatus arrangement was identical to that shown in figure 5, except for phonograph relocation from phonograph $\mathrm{c}$ to phonograph ("c" represents clinic, "s" represents school). The schoolroom did not contain the space above the dotted line in figure 5. Hence, in the schoolroom, raters were positioned at $R_{S 1}$ and $R_{S 2}$ and $\underline{E}$ and $\underline{E}_{S}$.

Ss sat on the floor in semicircle fashion around a 25-inch high table. Under the table was a phonograph speaker and a tape recorder. Hung from metal hooks, attached to the ceiling tile, was a $60 \times 74$ inch piece of cardboard containing $5 \times 7$ inch Texas Playing Cards. Texas Cards formed the display model in all treatments because they are easy to see from a distance. Regular $2 \frac{1}{2} \times 3 \frac{1}{2}$ inch playing cards

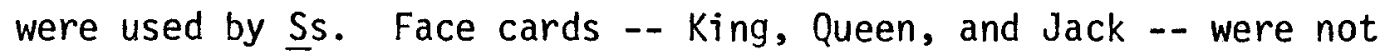
included in either the display or $\underline{S}$ cards, since it is likely that children are more familiar with numbers and might have difficulty in distinguishing between face cards.

Background sound was played under three of the four treatment conditions -- mood music (MM), art music (AM), and the voices of three adults reading aloud simultaneously from different textbooks (PT). The fourth condition was one of no sound (NS). In treatments MM and AM, sound was delivered via the portable stereo phonograph speaker. In the PT treatment, sound came from the tape recorder. The recorder, phonograph, and phonograph speaker were in full view of the $\underline{S}$ at all times, However, the phonograph was far removed, and the speaker and 
and recorder were in a nondistracting location under the table (see figure 5).

For each set of groups run during an afternoon at the school, or an evening at the clinic, E set the sound level. of the phonograph at an average peak of $64 \mathrm{~dB}$ (decibels) for mood music (range of $62 \mathrm{~dB}$ to $68 \mathrm{~dB}$ ) and $64 \mathrm{~dB}$ for art music (range of $50 \mathrm{~dB}$ to $70 \mathrm{~dB}$ ) using $\mathrm{a}$ General Radio Sound Level Meter, Type 1565-A, scale A-S. Likewise, the tape recorder sound level was adjusted to an average peak of $64 \mathrm{~dB}$ (range of $62 \mathrm{~dB}$ to $66 \mathrm{~dB}$ ). A normal speaking voice usually registers $60 \mathrm{~dB}$. In order to compensate for children's energetic shouts and cries so frequently heard when they play games, the higher peak of $64 \mathrm{~dB}$ was used.

\section{Procedure}

Training of raters. One male and two female volunteers from undergraduate psychology classes were trained to rate $\underline{S} s^{\prime}$ behavior on rating checklists prior to the study (see Appendix D). They met with $\underline{E}$ (male) on three occasions to discuss the rating categories and practice the rating routine. Each rater sat in the same location in the experimental room that he would occupy during the study, and rated the behavior of $\underline{E}$ and the other two raters who role played children. The rater was trained to carefully observe all actions of a $\underline{S}$ and check the cooperative and/or noncooperative behavior categories into which $\mathrm{S}^{\prime} \mathrm{s}$ behavior fell at the time of observation. If no category was appropriate, the rater checked the "other" category 
and briefly noted what the behavior was. E subsequently reviewed the raters' practice rating sheets and discussed any discrepancies between demonstrated behavior and checked categories with the raters individually. This training procedure was chosen because it not only closely paralleled the actual rating conditions, but also gave the raters a feeling for the types of behavior possible by having them take the role of the $\underline{S}$.

Unit of duration. The unit of duration for recording behavioral observations was alternate 5-second observation and 5-second recording intervals. During training and the experiment, raters listened to a tape recording of alternate one and two taps, produced by one coin striking another, while observing and recording behaviors. The taps were exactly the same number of seconds apart. Two taps signaled the start of an observation period, one tap the beginning of a sheet marking period. This assessment procedure used time as a means of randomly sampling observations of $\underline{S}^{\prime}$ behavior, and was later analyzed for number of cooperative and noncooperative behaviors emitted per minute under the different treatment conditions, as well as for inter-rater reliability (see Chapter III).

Procedural detail. Ss participating at the clinic were introduced to each other in the waiting room before the experiment began, in order to create a congenial atmosphere. Those participating at the school already were acquainted with each other. E led the $\underline{S}$ into the experimental room and told them to sit in a semicircle around 
the table in random order. After randomly giving two pieces of numbered cardboard tied together with string to each $\underline{S}$ to wear, he mentioned that people would be observing the game, but that their [children's] main concern was with the game. He hung the display, and then said the following:

Today we are going to play a game with cards. I will give each of you eight cards in a stack and place the stack face down in front of you. Please leave the cards just as I give them to you for now.

$\underline{E}$ distributed eight randomly ordered cards to each $\underline{S}$. After asking the groups to identify cards that he pointed to at random in the display, $\underline{E}$ instructed the $\underline{S} s$ as follows:

There are eight cards up here [pointing to the display modell. Each of you has eight cards. You all have some of the same kind of cards that you see up here, but none of you has all of them. When I tell you to begin, I want each of you to come up with a set of cards that looks just like the one you see up here. You will have to trade cards with your neighbors, because they have cards that you need, and you have cards that they need. Each person who gets one of each of these cards [pointing to the display model] will receive 15 M\&Ms. Any questions? Begin. Turn you cards over, and try to get all of the cards you see up here. Remember, you will have to trade cards with your neighbors.

E had randomly distributed exactly seven sets of cards matching the display, or a total of 56 cards. To form a set matching the display, a $\underline{S}$ had to ask one or more fellow $\underline{S}$ for cards that he needed. Maximum time allowed for task completion by the group was 10 minutes. During every session, two raters, randomly paired for each $\underline{S}$ group, sat in the experimental room (see figure 5 for location) with 
rating sheets attached to a clipboard. Both raters observed the same $\underline{S}$ at the same time, yet were told not to look at each other's sheets while rating. Order of $\underline{S}$ observation had been predetermined randomly, so that raters looked for the numbered cardboard, worn by $\underline{S} s$, that corresponded to the $\underline{S}$ number already marked on the rating sheet, and observed the $\underline{S}$ wearing that number during a given observation interval. 


\section{CHAPTER III}

\section{RESULTS}

Data for each experimental and control group were originally grouped in terms of number of cooperative and noncooperative responses recorded for each $\underline{S}$ for the duration of his or her group's performance. Subsequently, these data were transformed, for each $\underline{S}$, into cooperative and noncooperative responses per minute. Finally, a cooperative and noncooperative behavior sum was obtained by adding the individual cooperative and noncooperative totals in each group. In order to correct for the error in group response time, caused by the last two Ss finishing simultaneously in each group, only data from six of the seven $\underline{S}$ s in each group were used.

For reasons explained elsewhere (see Inter-Rater Reliability), two one-way analyses of variance were calculated for cooperative behavior, presented in Table 1 and 2 , and two for noncooperative behavior, found in Tables 3 and 4. A completely randomized design had been chosen in order to most effectively compare inter-group behavioral differences across all four treatment conditons. The amount of variance accounted for by the between group factor was no greater than that occurring by chance in all of the analyses performed. 
TABLE 1.

Summary of Analysis of Variance

for Cooperative Behavior* $I$

\begin{tabular}{lccccc}
\hline Source & SS & df & MS & F & $p$ \\
\hline Between & 3.59 & 13 & 1.20 & .37 & $>.20$ \\
Within & 51.97 & 16 & 3.25 & - & \\
Total & 55.56 & 19 & - & - & \\
\hline
\end{tabular}

* Tallying response residue as rater disagreement.

TABLE 2 .

Summary of Analysis of Variance

for Cooperative Behavior* II

\begin{tabular}{lrrrrr}
\hline Source & SS & df & MS & F & $p$ \\
\hline Between & 2.91 & 3 & .97 & .34 & $>.20$ \\
Within & 45.39 & 16 & 2.84 & - & \\
Total & 48.30 & 19 & - & - & \\
\hline
\end{tabular}

* Tallying response residue as neither rater disagreement nor agreement. 
TABLE 3.

Summary of Analysis of Variance

for Noncooperative Behavior * I

\begin{tabular}{lccccc}
\hline Source & SS & df & MS & $F$ & $p$ \\
\hline Between & 7.18 & 3 & 2.39 & 1.34 & $>.20$ \\
Within & 28.55 & 16 & 1.78 & - & \\
Total & 35.73 & 19 & - & - & \\
\hline
\end{tabular}

* Tallying response residue as rater disagreement.

TABLE 4 .

Summary of Analys is of Variance

for Noncooperative Behavior* II

\begin{tabular}{lrrrrr}
\hline Source & SS & df & MS & F & $p$ \\
\hline Between & 7.45 & 3 & 2.48 & 1.66 & $>.20$ \\
Within & 23.84 & 16 & 1.49 & - & \\
Total & 31.29 & 19 & - & -
\end{tabular}

* Tallying response residue as neither rater disagreement nor agreement. 
Looking at group totals for cooperative and noncooperative behavior (see Appendix B and C), a nonsignificant trend for noncooperative behavior to be greater under No Sound and People Talking conditions, and reduced under Art Music and Mood Music conditions, appears as predicted. Also, noncooperative behavior does tend to be increased and cooperative behavior decreased under the Art Music as compared to the Mood Music condition, though not significantly so. Unfortunately, the trend for more cooperation to be shown under music, as opposed to nonmusic conditions, is not as apparent.

Neither sex showed a noticeable difference in cooperative or noncooperative behaviors from the other. Age was not an influencing factor, and behavior scores obtained in the clinic and in the school showed little, if any, discrepancy for equivalent treatment conditions.

Figure 6 shows the mean total performance time for each set of five groups randomly assigned to a different treatment condition. Though not significantly different from one another, the performance times do show a downward progression, with nonmusic groups taking longer than music groups to complete the assigned task.

Inter-Rater Reliability

An inter-rater reliability ratio was calculated for each pair of raters on the basis of all groups that the pair jointly rated. Inter-rater agreement was defined as a mark from each rater under the same general category of "cooperative behaviors" or "noncooperative behaviors" for the same $\underline{S}$ observed during the same given interval. 
$6.6-$

$6.4-$

$6.2-$

$6.0-$

$5.8-$

$5.6-$

$5.4-$

$5.2-$

$5.0-$

$4.8-$

$4.6-$

$4.4-$

$4.2-$

$4.0-$

$3.8-$

$3.6-$

$3.4-$

$3.2-$

$3.0-$

$2.8-$

$2.6-$

$2.4-$

0

NS

PT

MM

AM

Figure 6. Mean Group Performance Time as a Function of Treatment Condition 
Disagreement occurred when the two marks were under different categories. There were several instances in which one rater had marked more cooperative behaviors than the other rater for the same $\underline{S}$ and observation interval. It was therefore decided to calculate two inter-rater reliability ratios -- one for which these extra responses (response residue) were tallied as disagreements, and the other for which they were tallied neither as disagreements nor as agreements. The formula used to calculate the ratio was the same as that used by Wodarski, et a1., (1972):

$$
\text { Inter-rater reliability ratio }=\frac{\text { Number of agreements }}{\begin{array}{l}
\text { Number of agreements } t \\
\text { Number of disagreements }
\end{array}}
$$

When response residue was treated as inter-rater disagreement, the mean inter-rater reliability ratio was .72; when disregarded, .87 . 


\section{CHAPTER IV}

\section{DISCUSSION}

Before the present study was conducted, an intensive review of relevant literature had produced little, if any, substantive bas is for hypothesis formulation and testing, simply because to the author's knowledge, no study investigating the effect of musical input on children's cooperative responding in a group problem solving context had even been published. Therefore, this investigation's orientation, of necessity, became one of exploration directed by procedural controls that were extrapolated from the literature resume. In no way was the experiment meant to validate or negate previous work done in either of the two related topic areas discussed earlier. If anything, it was hoped that some basis of integrating findings from both areas could be discovered. What follows is a discussion of why that basis was not found.

\section{Raters and Behavior Recording}

Certain elements of dependent variable measuring should be noted. Though not informed of E's hypotheses until the study had been concluded, assistants were unavoidably aware of each treatment condition under which they were rating, and even reported to $E$ various hypotheses they were forming about $\underline{S}$ behavior under different treatment conditions 
-- hypotheses which $\underline{E}$ neither confirmed nor denied. Also, during the course of observing 140 children, assistants might have been more alert at some times than at others, due to any of a number of physical and psychological variables in effect at the times of rating -- e.g., headache, anxiety related to upcoming exams. Nevertheless, assistants did receive thorough training in the rating procedure, and were able to maintain an inter-rater reliability coefficient of .72. More important, though, is the parallel between these rating conditions and those the children find operating in their everyday 1ives. Teachers, parents, and peers all observe these children interacting with other people, and subjectively classify the children's verbal and nonverbal patterns of interacting as cooperative and noncooperative. Such behavioral ratings, however, are always tinged to a degree by the teachers', peers', and parents' own prior interactions with the children. In this sense, ratings made by trained assistants with whom the children have never interacted with before, while procedurally closer to real life "rating" conditions than the Marble Pull Game (Madsen, 1971) or a telegraph key apparatus (Brotsky and Thomas, 1971), are also likely to contain less $\underline{S}$-influenced recorder bias.

It will be recalled that all observations were recorded on a rating form (see Appendix D). This form represents the culmination of work with two predecessor rating forms used in the pilot study. At each revision stage, the primary goal was to create a rating system 
with mutually exclusive, independent behavior categories (McNamara and MacDonough, 1972). In the process, some categories were added, some were pruned to essential words or phrases, while others were eliminated. To statistically establish a trend of increased interrater reliability with successive revisions would have required far more raters than the three used in the pilot study and the three in the current study, as well as more rating sessions. However, $E$ did note a change in the tenor of rater comments, regarding the rating form, from dissatisfaction with occasional overlapping categories during the initial pilot ratings, to satisfaction with the final classificatory scheme that seemed to cover most behaviors observed by the early part of the current study.

It could be that a rater's task would be simplified if he were responsible for fewer behavioral categories per observation interval. Conceivably, seven rater pairs could be employed to rate all $\underline{S}$ groups, where each pair member would be responsible for checking either "observed" or "not observed" for the one behavior category assigned to that pair for a given group. Given the same 20 rating sessions, raters would have to be permanently paired for the experiment's duration in order to obtain reliability coefficients based on more than just one rating session per pair. Category assignment to rater pairs could be done on a random or rotating basis. However, the logistics of assembling 14 raters for 20 sessions, combined with the mechanics of meaningfully integrating seven sets of reliability 
ratios (probably confounded by observer drift) seems an unwieldy task. Perhaps simply revising and reusing behavioral categories until obtaining a set that produces the highest, overall agreement ratio between raters is the soundest alternative.

\section{Subjects}

Another influencing factor concerns the children operating in the immediate experimental environment. Motivation, experimental setting, and individual differences were all joint contributors to ultimate behavioral output, and determining the importance of each relative to the others, as well as to the dependent variable, would probably challenge even the most devout factor analysis disciple.

Motivation, as judged by $\underline{E}$, seemed adequate for most $\underline{S}$ groups. If there was a difference in desire to actively participate between pilot $\underline{S} s$, who were each given $\$ 1$ beside the M\&Ms, and current $\underline{S} s$ who merely received M\&Ms, it was not apparent. Specifically regarding the reinforcing value of M\&Ms, however, comments like, "Oh boy, M\&Ms," and "I really like M\&Ms" seemed to occur often among the younger children. 0lder sophisticates either expressed no opinion of the M\&Ms offered, or occasionally shrugged their shoulders to indicate a "so what else is new" situation appraisal. Hence, motivation to

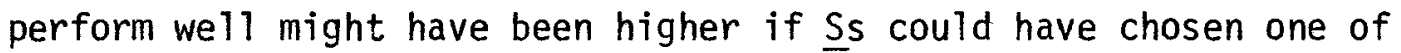
several to-be-earned reinforcers before beginning the card task. Also, task material could have been made more relevant to Ss' everyday environment in attempt to maximize motivation. For example, 
cards depicting classroom scenes, and even including names of teachers and fellow pupils could have been substituted for the ordinary playing cards.

As noted in Chapter II, somewhat less than half of the $\underline{S}$ groups were at the University of Montana's Clinical Psychology Center, while the rest participated at one of the local parochial schools. Physical arrangement of apparatus was very similar in both settings, and each experimental room was sufficiently removed from distracting stimuli -visual and auditory. If any factor might have produced more cooperative responding in one setting than the other, it could have been the pre-experimental familiarity with the experimental room (minus the apparatus) of school $\mathrm{Ss}$, as opposed to clinic $\underline{\mathrm{Ss}}$ ' total unfamiliarity with their experimental room. It could be argued that a totally novel environment could have inhibited individualistic tendencies to remain aloof, and facilitated conformity to the group norm, whether cooperative or noncooperative, in order to gain peer support and thereby increase one's sense of security. While this line of reasoning probably held for a few $\underline{S} s$, it did not for the majority, since the average spread of individual cooperative and noncooperative responses per school group was not noticeably larger than that of clinic groups. Also, the fact that more school Ss were familiar with each other before participating in the study than were clinic Ss seemed to have had little or no bearing upon total performance time per group, thus questioning the relevance of pre-experimental acquaintanceships among $\underline{S}$ s to experimental findings. 
Finally, it does not seem unreasonable to postulate a degree of correlation between the amount of cooperative behavior displayed and individual difference factors. Current level of intellectual functioning, prior social reinforcement history, introversion-extroversion tendencies, and gross and fine visual-motor coordination all combined to form seven unique bundles of respondent potential in all $\underline{S}$ groups. Due to the extremely large within-group variance obtained for both pilot and current study $\underline{S}$, the author feels confident that the results were undoubtedly influenced by any one or a combination of these variables, and that future research will have to reliably measure and group $\underline{S}$ s on these variables in such a way as to sizeably reduce this within-Ss error term. The task may not be as formidable as it sounds, since scales, of respectable validity and reliability, already exist for all these variables except prior social reinforcement history, which could be assessed on the basis of peer, teacher, and parent interviews.

\section{Experimental Task}

Undoubtedly, cooperative problem solving in this study depended on Ss ability to attend to task-relevant stimuli and eschew, for the time being, other stimuli not directly contributing to goal attainment. It could be that the amount of concentration called for in trading cards with one's neighbors, in order to complete a given set of cards, was not enough for measuring the effect of background music on inter- $\underline{S}$ responding. By increasing the level of task complexity, future research 
could more directly focus upon concentration in children as a function of sound input. Another possibility might be to pair group-task responding with mood music and individual-task responding with art music (or vice-versa) at initially low levels of task complexity. If conditioning is established, each music condition will facilitate - performance in only that type of task with which it (music) had been paired. Generalization to higher complexity levels could be tested by comparing group and individual performance times with those of placebo control $\underline{S} s$ who receive no sound input during their low complexity level sessions.

Final conclusions regarding the effects of music on cooperative problem solving in children should not be made at this time. It may be that children's sensory processing of art and mood music is at a less complex level than that for adults, and therefore require repeated exposure to both types of music, under a variety of conditions, before the processing can be refined. Consideration and implementation of suggested improvements in methodology should increase the probability of more clear-cut results in future research. 


\section{CHAPTER $V$}

\section{SUMMARY}

One hundred and forty children, six to nine years of age, were randomiy assigned to groups of seven and given a card-matching task to complete. Group members were required to trade cards with one another in order to obtain a set identical to a display model under one of four conditions of sound input: mood music, art music, people talking, and no sound. In return for completing his or her set, each $\underline{S}$ received 15 M\&Ms. Trained observers used a time-sampling technique to record cooperative and noncooperative responding on a behavior checklist.

The study's results found no statistical difference between the number of cooperative responses emitted under music conditions and that under people talking and no sound conditions, nor between amount of cooperative responding under art music as opposed to mood music. Likewise, the number of noncooperative responses displayed under treatment conditions did not vary more than would be expected by chance alone. A nonsignificant trend toward more noncooperative responses being shown under music than no music conditions was noted, as was one of increased cooperative responding under mood music over that of art music. Reasons for lack of significant treatment effects are offered, and implications for future research are discussed. 


\section{REFERENCES}

Allen, K. E., Hart, B., Buell, J. S., Harris, F. R., and Wolf, M. M. Effects of social reinforcement on isolate behavior of a nursery school child. Child Development, 1964, 35, 511-518.

Altman, K. Effects of cooperative response acquisition on social behavior during free-play. Journal of Experimental Child Psychology, 1971, 12, 387-395.

Azrin, N. H., and Lindsley, 0. R. The reinforcement of cooperation between children. Journal of Abnormal and Social Psychology, 1956, 52, 100-102.

Brotsky, S. J., and Thomas, K. Cooperative behavior in preschool children. Psychonomic Science, 1967, 9, 337-338.

Bushel1, D., Wrobel, P. A., and Michaelis, M. L. Applying "group" contingencies to the classroom study behavior of preschool children. Journal of Applied Behavior Analys is, 1968, 1, 55-61.

Diephouse, D. A. Music therapy in a child psychiatry clinic school. American Music Teacher, 1968, 32, 40.

Diephouse, J. W. Music therapy: A valuable adjunct to psychotherapy with children. Psychiatric Quarterly Supplement, 1968, 42, $75-85$.

Doland, D. J., and Adelberg, K. The learning of sharing behavior. Child Development, 1967, 38, 695-700.

Dryer, J., and Dix, J. Reaching the blind children through music therapy. Journal of Emotional Education, 1968, 8, 202-211.

Fischer, W. F. Sharing in preschool children as a function of amount and type of reinforcement. Genetic Psychology Monographs, 1963, $\underline{68}, 215-245$.

Freeburne, D. M., and Fleischer, M. S. The effect of music distraction upon reading rate and comprehension. Journal of Educational Psychology, 1952, 43, 101-109. 
Gatewood, E. L. An experiment in the use of music in an architectural drafting room. Journal of Applied Psychology, 1921, $5,350-358$.

Greenberg, R. P., and Fisher, S. Some differential effects of music on projective and structured psychological tests. Psychological Reports, 1971, 28, 817-818.

Hake, D. F., and Vukelich, R. A classification and review of cooperation procedures. Journal of the Experimental Analys is of Behavior, $1972, \underline{18}, 333-343$.

Handlon, B. J., and Gross, P. The development of sharing behavior. Journal of Abnormal and Social Psychology, 1959, 59, 425-428.

Hannah, B. H. Cooperation and competition in the classroom: Effects of the degree of similarity of the task on behavior in children's groups. Dissertation Abstracts International, 1971, 31, (12A), 6402 .

Harford, T., and Cutter, H. S. Cooperation among negro and white boys and girls. Psychological Reports, 1966, 18, 818.

Hart, B. M., Reynolds, N. J., Baer, D. M., Brawley, E. R., and Harris, F. R. Effects of contingent and non-contingent social reinforcement on the cooperative play of a preschool child. Journal of Applied Behavior Analysis, 1968,1,73-76.

Hops, $H$. The school psychologist as a behavior management consultant in a special class setting. Journal of School Psychology, 1971, 9, 473-483.

Jacoby, J. Work music and morale: A neglected but important relationship. Personnel Journal, 1968, 47, 882-886.

Jensen, M. B. The influence of jazz and dirge music upon speed and accuracy of typing. Journal of Educational Psychology, 1931 , 22, 458-462.

Kagan, S., and Madsen, M. C. Cooperation and competition of Mexican, Mexican-American, and Anglo-American children of two ages under four instructional sets. Developmental Psychology, 1971, 5, 32-39.

Kagan, S., and Madsen, M. S. Experimental analyses of cooperation and competition of Anglo-American and Mexican children. Developmental Psychology, 1972, 6 , 49-59. 
Katz, L. F. The prediction of cooperative behavior in children through the use of sociometric scores. Dissertation Abstracts, 1966, 27, (4B), 1280.

Lathom, W. Concepts of information theory and their relationship to mus ic therapy. Journal of Music Therapy, 1971, 8, 111-116.

Luchins, A. S., and Luchins, E. H. Cooperativeness of task in relation to discovery of source of contradictory communications. The Journal of General Psychology, 1957, 56, 159-178.

McClintock, C. G., and Nuttin, J. M. Development of competitive game behavior in children across two cultures. Journal of Experimental Social Psychology, 1969, 5, 203-218.

McKee, J. P., and Leader, F. B. The relationship of socioeconomic status and aggression to the competitive behavior of preschool children. Child Development, 1955, 26,135-142.

McNamara, J. R., and MacDonough, T. Some methodological considerations in the design and implementation of behavior therapy research. Behavior Therapy, 1972, 3 , 361-378.

Madsen, M. C. Cooperative and competitive motivation of children in three Mexican sub-cultures. Psychological Reports, 1967, 20, 13071320.

Madsen, M. C. Developmental and cross-cultural differences in the cooperative and competitive behavior of young children. Journal of Cross-Cultural Psychology, 1971, 2, 365-371.

Madsen, M. C., and Shapira, A. Cooperative and competitive behavior of urban Afro-American, Anglo-American, Mexican-American, and Mexican village children. Developmental Psychology, 1970, $\underline{3}$, 16-20.

Meichenbaum, D. H., Bowers, K. S., and Ross, R. R. A behavioral analysis of teacher expectancy effect. Journal of Personality and Social Psychology, 1969, 13, 306-316.

Miller, A. G., and Thomas, R. Cooperation and competition among Blackfoot Indian and urban Canadian children. Child Development, $1972, \underline{43}, 1104-1110$.

Mithaug, D. E. The development of cooperation in alternative task situations. Journal of Experimental Child Psychology, 1969, 8, 443-460. 
Mithaug, D. E., and Burgess, R. L. The effects of different reinforcement contingencies in the development of social cooperation. Journal of Experimental Child Psychology, 1968, $\underline{6}$, $402-426$.

Mussulman, J. The use of music: An introduction to music and music life in contemporary western culture, 1974, in press.

Nelson, L. L. The development of cooperation and competition in children from ages five to ten years old: Effects of sex, situational determinants, and prior experiences. Dissertation Abstracts International, 1971, 31, (7-B), 4368.

Nelson, L., and Madsen, M. C. Cooperation and competition in fouryear-olds as a function of reward contingency and subculture. Developmental Psychology, 1969, 1, 340-344.

Owens, K. L. Competition in children as a function of age, race, sex, and socio-economic status. Dissertation Abstracts International, $1970,30,(8-B), 3873$.

Peters, R. W., and Torrance, E. P. Dyadic interaction of preschool children and performance on a construction task. Psychological Reports, 1972, 30, 747-750.

Pike, A. The phenomenological analysis and description of musical experience. Journal of Research in Music Education, 1967, 15, 316-319.

(a)

Pike, A. The theory of unconscious perception in music: A Phenomenological criticism. The Journal of Aesthetics and Art Criticism, 1967, 25, 395-400. (b)

Presbie, R. J., and Kanareff, V. T. Sharing in children as a function of the number of sharees and reciprocity. The Journal of Genetic Psychology, 1970, 116, 37-44.

Rieber, M. The effect of music on the activity level of children. Psychonomic Science, 1965, 3, 325-326.

Scott, T. J. The use of music to reduce hyperactivity in children. American Journal of Orthopsychiatry, 1970, 40,677-680.

Shapira, A. Competition, cooperation and conformity among city and kibbutz children in Israel. Dissertation Abstracts International, $1971,31,(7-A), 3641-3642$. 
Shapira, A., and Madsen, M. C. Cooperative and competitive behavior of kibbutz and urban children in Israel. Child Development, $1969,40,609-617$.

Smith, H. C. Music in relation to employee attitudes, piece-work, production, and industrial accidents. Applied Psychological Monographs, 1947, 14.

Staub, E., and Sherk, L. Need for approval, children's sharing behavior, and reciprocity in sharing. Child Development, 1970, 41, 243-252.

Steele, A. L. Programmed use of music to alter uncooperative problem behavior. Journal of Music Therapy, 1968, 5, 103-107.

Stevens, E., and Clark, F. Music therapy in the treatment of autistic children. Journal of Mus ic Therapy, 1969, $6,98-104$.

Sugarman, P. A musical program for emotional high blood pressure. In E. Podolsky (Ed.), Music Therapy. New York: Philosophical Library, 1954, 151-152.

Ugure1-Semin, R. Moral behavior and moral judgment of children. Journal of Abnormal and Social Psychology, 1952, 47, 463-474.

Wasik, B. H., Senn, S. K., and Epachin, A. Cooperation and sharing behavior among culturally deprived preschool children. Psychonomic Science, $1969,17,371-372$.

Weidenfeller, E. W., and Zimny, G. H. Effects of music upon GSR of depressives and schizophrenics. Journal of Abnormal and Social Psychology, 1962, 64, 307-312.

Weigle, V. Functional music: A therapeutic tool in working with the mentally retarded. American Journal of Mental Deficiency, $1959,63,672-678$.

Weingold, H. P., and Webster, R. L. Effects of punishment on a cooperative behavior in children. Child Development, 1964, $\underline{35}, 1211-1216$.

Wodarski, J. S., Hamblin, R. L., Buckholdt, D. R., and Ferritor, D. E. The effects of low performance group and individual contingencies on cooperative behaviors exhibited by fifth graders. The Psychological Record, 1972, 22, 359-368. 
Wright, B. A. Altruism in children and the perceived conduct of others. Journal of Abnormal and Social Psychology, 1942, 37, 218-233.

Zimny, G. H., and Weidenfeller; E. W. Effects of music upon GSR of children. Child Development, 1962, 33, 897-896.

Zimny, G. H., and Weidenfeller, E. W. Effects of music upon GSR and heart rate. American Journal of Psychology, 1963, 76, 311-314.

Zwier, M. D. Psychosocial factors in communication among elementary school children. Dissertation Abstracts, 1965, 25 (11), 68046805 . 


\section{APPENDIXES}

61 
APPENDIX A

Pilot Data Summary

Cooperative Responses per Treatment

$\begin{array}{llll}H & \text { PT } & \text { NH } & \text { NS }\end{array}$

$\begin{array}{crrrrr}\text { Group } & & & & & \\ 1 & 20 & 6 & 22 & 1 & 49 \\ 2 & 18 & 12 & 26 & 15 & 71 \\ \text { Total } & 38 & 18 & 48 & 16 & 120\end{array}$

Noncooperative Responses per Treatment

$\begin{array}{lllll}H & \text { PT } & \text { NH } & \text { NS } & \text { Total }\end{array}$

$\begin{array}{crrrrr}\text { Group } & & & & & \\ 1 & 9 & 13 & 20 & 59 & 101 \\ 2 & 11 & 15 & 2 & 29 & 57\end{array}$

$\begin{array}{llllll}\text { Total } & 20 & 28 & 22 & 88 & 158\end{array}$

Cooperative Responses per Treatment Minute

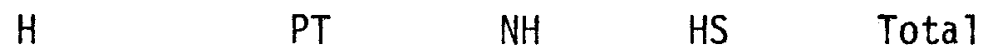

$\begin{array}{crrrrr}\begin{array}{c}\text { Group } \\ 3\end{array} & 4.67 & 2.63 & 2.11 & 1.68 & 11.09 \\ 4 & 2.99 & 5.08 & 3.07 & 1.60 & 12.74 \\ 5 & 6.58 & 5.47 & 0.84 & 4.55 & 17.44 \\ \text { Total } & 14.24 & 13.18 & 6.02 & 7.83 & 41.27\end{array}$

Noncooperative Responses per Treatment Minute

$\begin{array}{lllll}H & \text { PT } & \text { NH } & \text { HS } & \text { Total }\end{array}$

\begin{tabular}{crrrrr}
$\begin{array}{c}\text { Group } \\
3\end{array}$ & 9.56 & 9.53 & 9.71 & 10.24 & 39.04 \\
4 & 9.34 & 8.53 & 5.81 & 8.16 & 31.84 \\
5 & 6.17 & 5.81 & 6.95 & 7.44 & 26.37 \\
Total & 25.07 & 23.87 & 22.47 & 25.84 & 97.25 \\
\hline
\end{tabular}


APPENDIX B

Current Data Summary

Including

Residue Ratings

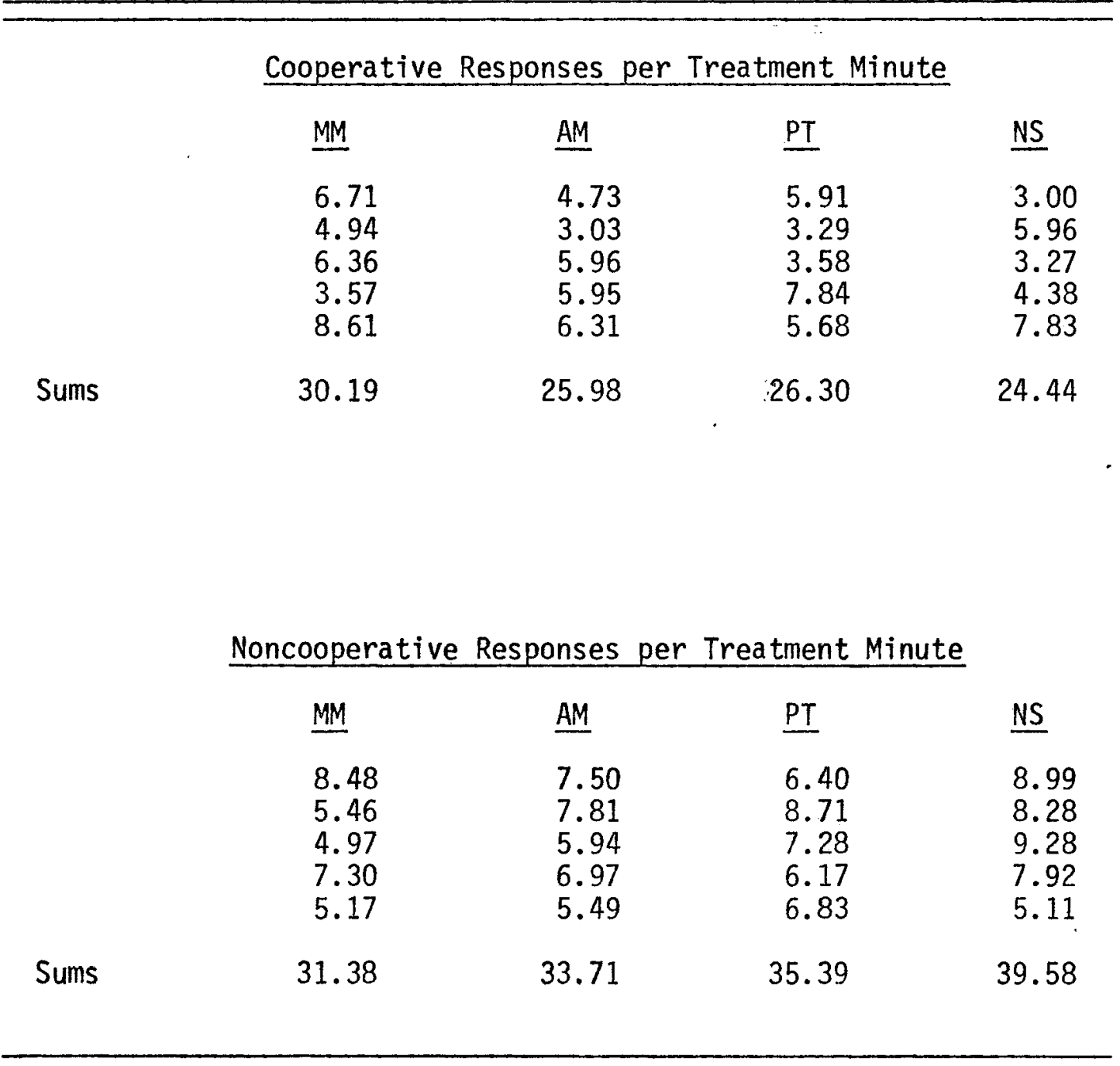


APPENDIX C

Current Data Summary

Excluding

Residue Ratings

Cooperative Responses per Treatment Minute

MM $\quad \underline{\text { AM }} \quad \underline{\text { PT }} \quad \underline{\text { NS }}$

$\begin{array}{llll}5.32 & 3.28 & 5.27 & 2.01 \\ 4.14 & 2.26 & 2.71 & 4.94 \\ 5.39 & 4.73 & 3.29 & 2.14 \\ 2.98 & 4.42 & 6.73 & 4.10 \\ 6.87 & 5.20 & 5.11 & 7.52\end{array}$

$\begin{array}{lllll}\text { Sums } & 24.70 & 19.89 & 23.11 & 20.71\end{array}$

Noncooperative Responses per Treatment Minute

$\begin{array}{llll}\text { MM } & \text { AM } & \text { PT } & \text { NS } \\ 7.89 & 7.10 & 6.40 & 8.57 \\ 5.07 & 7.30 & 8.13 & 7.40 \\ 4.97 & 5.45 & 7.14 & 9.00 \\ 6.71 & 5.95 & 5.42 & 7.50 \\ 4.92 & 5.21 & 6.64 & 5.11\end{array}$

$\begin{array}{lllll}\text { Sums } & 29.56 & 31.01 & 33.73 & 37.58\end{array}$


APPENDIX D

Group

Cooperative behaviors:

Ss

Gives or takes cards

he (she) needs

Manuaily assists

other $\underline{S}$

Verbally assists or

talks to other $\underline{\text { Ss }}$

S

Gives or takes cards he (she) needs

Manually assists

other Ss

Verbally assists or talks to other Ss

$\underline{S}$

Gives or takes cards he (she) needs

Manually assists

other S

Verbally assists or talks to other $\underline{S}$ s
Treatment

Noncooperative behaviors:

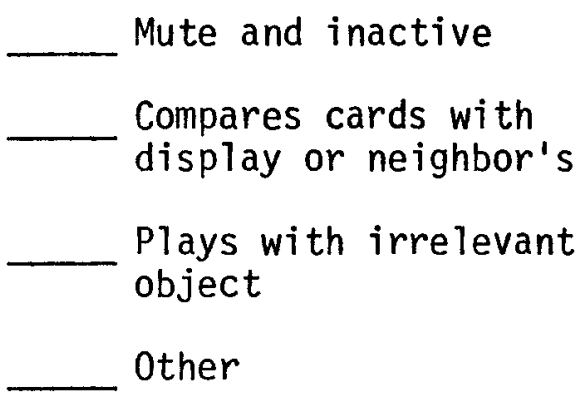

Mute and inactive Compares cards with display or neighbor's Plays with irrelevant object other

Mute and inactive Compares cards with display or neighbor's Plays with irrelevant object

Other 